\title{
The ever expanding issue of later life mental health
}

\author{
K. Glynn ${ }^{1}$ and J. Lyne ${ }^{2, *}$ \\ ${ }^{1}$ North Dublin Mental Health Services, Ashlin Centre, Beaumont Road, Dublin 9, Ireland \\ 2 Royal College of Surgeons in Ireland and North Dublin Mental Health Services, Ashlin Centre, Beaumont Road, Dublin 9, Ireland
}

An ageing global population represents one of the most significant social transformations for the decades ahead. The world's older population is growing at an unprecedented rate which will inevitably require an increased focus on the key issues affecting this population. From a healthcare perspective, the high prevalence of dementia and delirium among this population emphasises the importance of providing high-quality care for these conditions. In the current issue of Irish Journal of Psychological Medicine we focus on these themes as well as other issues such as the well-being of older adults.

The Lancet Commission on Dementia Prevention, Intervention and Care report was widely publicised on its release as providing hope for potentially reducing the prevalence of dementia through addressing modifiable risk factors (Livingston et al., 2017). In their editorial in the current issue, several of the Lancet Commission report authors take us through the key report findings and recommendations (Orgeta et al., pp. 85-88), chief among which are addressing nine modifiable risk factors for developing dementia. Collectively these risk factors are estimated to account for $35 \%$ of the population risk and the authors suggest that preventive strategies targeting these risk factors have the potential to delay or prevent dementia. Prevention strategies for consideration include increased public education, targeting vascular risk factors (hypertension, diabetes, obesity, smoking), and managing depression, social isolation and hearing impairment. The editorial also provides a useful summary of biopsychosocial interventions for managing dementia.

One area of research that shows promise in the treatment of dementia is cognitive rehabilitation, with previous research indicating that it can improve goal performance and enhance well-being in the early stages of the disease. In their Pilot study, Kelly et al. (pp. 105-119) show that 8 weeks of 60-90 minutes weekly sessions improved goal performance, goal satisfaction and quality of life in a small sample of individuals with early Alzheimer's disease. Larger-scale randomised controlled trials are now needed to replicate these findings.

* Address for correspondence: J. Lyne, Royal College of Surgeons in Ireland and North Dublin Mental Health Services, Ashlin Centre, Beaumont Road, Dublin 9, Ireland.

(Email: john.paul.lyne@gmail.com)
For adequate resourcing and healthcare service provision, it is essential that we have accurate estimates of dementia prevalence, both in Ireland and internationally. Pierse et al. (pp. 129-137) apply rates from prominent international studies to Irish census data to determine dementia prevalence in Ireland. They estimate dementia prevalence of up to 55266 in the Republic of Ireland, with a rising incidence of at least 7752 new dementia cases per year. The authors also estimate that 11175 individuals with dementia are living at home in the community with serious functional impairment. These findings are echoed in O'Brien's study (pp. 139-144), which shows a high proportion of those in receipt of state-funded homecare packages may have either suspected cognitive impairment $(8.7 \%)$ or dementia $(37.1 \%)$. Taken together, these studies point to the large health service provision needed in this vulnerable group, especially as the figures for those with cognitive impairment are expected to increase in the coming years.

Delirium is a frequently encountered cause of cognitive impairment in the elderly population, particularly in healthcare settings. Despite its high frequency, the diagnosis and treatment of delirium remain a challenge. This is illustrated by Wilson et al. (pp. 121-127) who report that delirium can often remain undetected in nursing homes. The study found that detection of delirium improved following the introduction of a clinical nurse specialist who provided support and training for nursing home staff. Also in this issue, O'Regan et al. (pp. 89-98) showed that a focused 2-hour intervention for psychiatrists run by delirium experts had a significant positive impact on their knowledge of key aspects of delirium care. Taken together, these studies highlight the importance of continued training and professional development for healthcare staff to ensure that complex clinical challenges can be addressed effectively.

Other highlights of the current issue include an audit by Sweeney et al. (pp. 145-151) of an Irish memory clinic against UK MSNAP guidelines, providing useful pointers towards areas of development and improvement of these clinics. Duffy et al. (pp. 99-104) find that the economic recession has had a negative effect on the subjective well-being in older adults, serving as an important reminder of the effects of economic hardship among older population groups. Finally, 
Reid's (pp. 153-155) perspective piece gives a personable account of the benefits offered by therapy dogs in reducing agitation, depression and loneliness among vulnerable residents in therapeutic settings.

\section{Conflict of interest}

K.G. and J.L. have no conflicts of interest to disclose.

\section{Reference}

Livingston G, Sommerlad A, Orgeta V, Costafreda SG, Huntley J, Ames D, Ballard C, Banerjee S, Burns A, Cohen-Mansfield J, Cooper C, Fox N, Gitlin LN, Howard R, Kales HC, Larson EB, Ritchie K, Rockwood K, Sampson EL, Samus Q, Schneider LS, Selbaek G, Teri L, Mukadam N, (2017). Dementia prevention, intervention, and care. Lancet. 390, 2673-2734. 